\title{
$\Sigma \mathrm{YN} \Theta \mathrm{E} \Omega$.
}

\section{PHYTOGRAPHIÆ SACR $Æ$ \\ G E N ER A L I S.}

Pars Practica fexta.

Quam
AuxilIANTE DEO
PRASIDE

JOHANNE GESNERO, Med. D. Phyl. \& Math. Prof.

ACADEM. IMPERIALIS NATUR E CURIOSO. RUM GERMANIC E ET RUSSICE PETROPOLITANA ATQUE SOCIET. REGIAR. SCIENTIAR. BER OLINENSIS SUECIC $Æ$ UPSAL. GOETTING. BOTANIC E FLOREN. TIN $\approx$ ET PHYSICO-MEDICÆ BASIL. MEMBRO.

Pro confequendo Examine Pbilofopbico Defendent

$\begin{array}{ll}\text { CONRADUS } & \text { OERIUS. } \\ \text { LEONHARDUS } & \text { WIRZIUS. } \\ \text { CONRADUS } & \text { NYSCHELERUS. } \\ \text { SALOMON } & \text { OBERMANNUS. } \\ \text { HENRICUS } & \text { HEFELINUS. }\end{array}$

$$
\text { Ad diem XाI" Febri }
$$

\section{$T U R I C I$}

EX OFFICINA GESSNERIANA. Anno MDCCLXV 
Digitized by the Internet Archive in 2019 with funding from Wellcome Library 


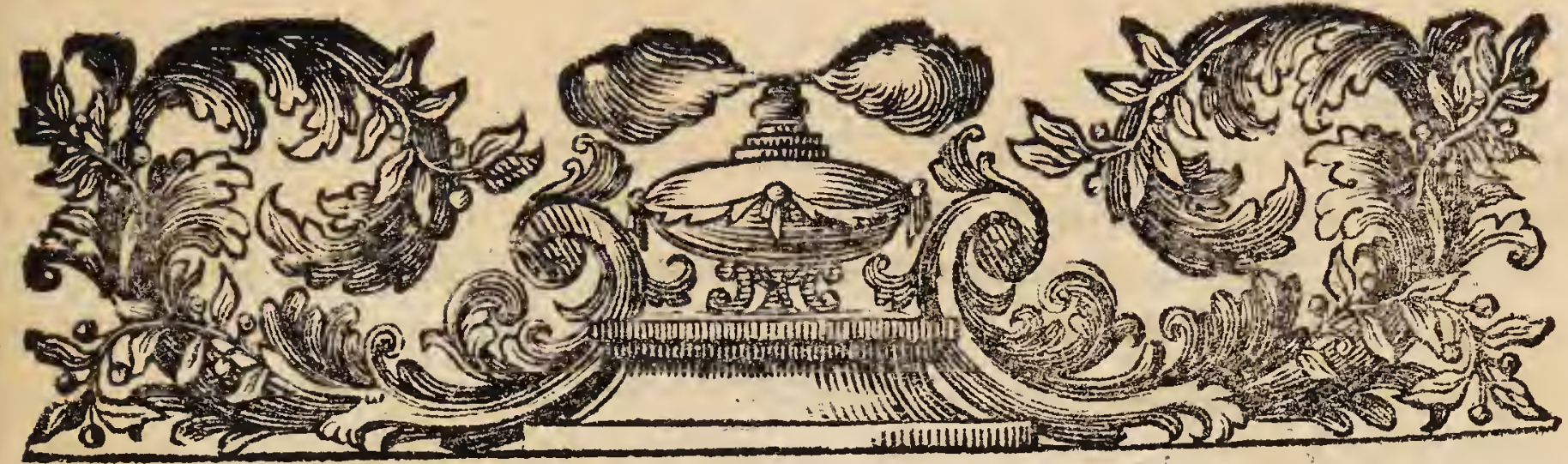

$\Sigma \Upsilon N \oplus E \Omega$.

\section{PHYTOGRAPHIE SACRE GENERALIS. \\ Pars Practica fexta. \\ I.}

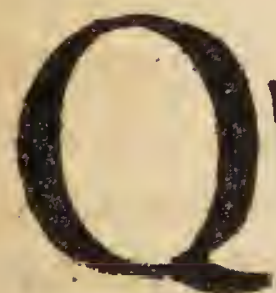

Uam bene, Optimus Maximus Rerum Conditor generi Nexus cu humano in difponenda Creaturarum omnium univerfi- praceden tate confuluerit, id omnia Naturæ Opera evidentifime nea $^{2}$ teftantur. Idemque luculentiffime ex Regno Vegetabili agnofcitur, in quo omnia ad perfectionem Univerfi mirifica confenfione confpirant. Id ipfum hactenus ex fabrica, functionibus, \& utilitatibus Plantarum aliquot præmiffis Differtationibus demonftrare fum annifus, pergo in exhibendis ufibus multivariis, quos Vegetabilia in Ædificiis \& Vitæ commoditatibus hominibus praftanto

II.

Qui experiri velit eximium Vegetabilium ufum, quem cum in ædificiis, tum in variis Vitæ commoditatibus præbeant, modo fecum tacisa cogitatione reputet, quxnam ex abfentia eorum, qux fecum tacita cogitatione reputet, quxnam ex abfentia eorum, qux adificiis
ex Ligno \& Vegetabilibus in inftructa domo \& oeconomia haben- commod tur, nafcitura fint incommoda, defectus, hiatus, lacunz.

Si ablit A. 2 lignum, 
lignum ; deftituta erit fimul domus plerumque fuis januis, portis, rcalis, parieribus, contignationibus, tectis, fubltructione, utenflium parte multò maximâ, men la, fcamnis, fubfelliis \& Inftrumen. tis plerisque; Foco, quo calorem foveat, alimenta paret; alimentorum, potulentorum, Medicamentorum, Veltimentorum parte prxcipua carebit. Defiderabit omne ferè chartarum genus, oleum, fuliginis atrum pigmentum, Cineres, Salia, lixiviofa, fapones, \& mille genus alia, qux vegetabilia in varios Ufus adaptata \& præparata fuppeditare folent. Quantum plantx vivx ad tegmen, umbram, tutelam conferant, if fuperiori Differtatione expofui ; reflant exponenda commoda multò numerofiora, quæ ex refectis \& adaptatis plantis obcinere licet. Patebit id luculentius, fi ea, qux

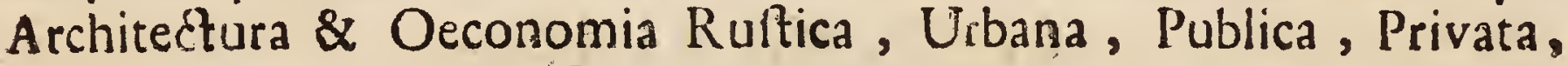
Civilis, Sacra, Navalis \& plurimx Artes ex Regno Vegetabili depromunt, generatim exponantur. Deinde oltendatur, quanta ubertate \& varietate fingula, qua cuique fcopo omnium optime convenire poffunt, five ea ad firmitatem, five ad commoditatem, five ad ornatum pertinent, in Vegetabilibus magno numero inveniantur. Quod ipfum primo demonftrabimus in Lignis variis ad Edificia in Sacris Literis memorata adhibitis, deinde per univerfum Regni Vegetabilis ambitum excurrentes Plantarum varias Claffes perluftrabimus, qux fuam hic Symbolam conferunt, ut pateat, quam late, quam copiofè Divinus Rerum omnium Largitor ubivis Gentium fua bona difpenfarit.

\section{III.}

rchite?tuinitia,

Architectura ædificandi ars, uti artes pleræque aliæ ex Infantia quafi \& parvis initiis ad adolefcentiam \& Virilem ætatem lente \& infenfibili velut incremento ad magnam perfectionem fummumque Faftigium perducta eft. Partim quod primorum hominum long 2 vitas, ad aliquot frcula fe extendens, longam multorum annorum experientiam, optimam Rerum magiftram, fuppeditabat, qux ex juvantibus \& nocentibus pulchre docebat \& difcebat qux vitanda, qux confectanda ad tutam commodamque vitam agendam. Partim quod Architecturæ perfectiflima exempla cum in iplis Naturx Operibus oculis \& menti fubjicerentur, tum \& fpecimina maximè 
illuftria a Deo ipro in Arca Noachi, in Tabernaculo, \& in Templo Salomoneo fuerint propofita, qux omnis perfectionis \& venuftatis Archicectonica veluci normam fuppeditare poffent.

Primevi itaque homines ardores Solis, Brumale frigus, pluviæ \& venti incommoda evitaturi fibi de latibulis, Tectis, ædibus profpiciebant. Atque ipla vivx Arbores fponte veluti tutelam ob. culerunt, coeperunt itaque de Fronde facere tecta, tum Hirundinum modo loca ex luto \& virgultis conftruere, quæ fubirent. Dein erectis ex arboreis ramis, furcis, interpofitis vel inter textis virgultis isciemque lito firmatis habitacula parabant, qux fronde vel arundine tegebant, aut fcandalis robufteis vel ftramentis. (a) Vel \& impofitis alternatim arboribus ad angulos rectos coagmentatis rectangulum adificium extruebant facile tecto muniendum. Ipfi quoque Arborum Trunci cylindrici primum \& fimpliciffimum columnarum genus fuppeditabant, thi ex angulis bafeos rectangula erecti \& per transverfas conjundti fimiles priori ædes exhibebant. Alii ftipites inter fe religantes metas efficiebant, quas arundinibus \& Itramentis tegentes, exaggerabant fupra habitationes maximos grumos e terra. Ita hiemes calidiflimas, æltates frigidiffimas efficrunt Tectorum ope. Nonnulli ex ulva paluftri componebant Tuguria tecta. Vel ex bafi rectangula, circulari, multangulari, virgx erigebantur, quæ fpatium includant parallelipipedum vel cylindricum, aut prismaticum Tecto pyramidali, vel conico, vel hamisphrrico vel cuneato contegendum. Vel exdem virgx ad angulum recto minorem inclinatæ in fummitate mutuo nexu firma. tæ Cafarum \& Tentoriorum varias fpecies efformabant, pannis, pellicibus, arundine, vel Stramine aut Scandalis tegendas. $\mathrm{Ha}$ rum apud varias Gentes varietatem fummatim tradit Cl. Hallen(b). Neque a fimpliciorum ædificiorum fabrica abludunt Calæ Alpicolarum Helvetix noltræ \& Lapponix, Gafx alpinæ, deferibente A 3

Celeb.

(a) Vitruv. Archited. L. X. Amftel. 1649. fol. Lib.II. C.I. pag. 18.

(b) Werkfátte ber Růnften, ober neue Sunft, Spiftorie, Tom. III Brandent. und fripzig 1764,4 , p. 3. 
Celcb. SCHezichzer o (c), parietes ex Trabibus lignis non vel ruditer exafciatis, invicemque fuper impolitis conftant; \& fxpe corricibus abiegnis veftiuntur ad arcendas Tempeftatum injurias. Tectum ex Scandalis ligneis per Saxa aggravatis, ne ab ipro Ventorum impetu disjiciantur. Tabulatum Terra nuda vel Corticibus abietum pinuumque ftrata. Haud longe a Cafa eft fimilis Architecturæ ftabulum, five Cafa Vaccaria. Ucenfilia lignea. Scamnum monopodium, cui Senna (Paftor) infider. Vafa muldrum, amphoræ, Colatorium, Fulcra \& fuftentacula varia, alveus, ar= culx, Patell $x$ varix, Dolium, vel Cylindrus butyro conficiendo deflinatus. (d) Lapponis Cafa feu Tuguriolum \& Domus gaudet forma pyramidali, hexagona apice truncato, altitudinis humanx, latitudinis triplo majoris. In Alpibus eft tentorium, conftans trabibus feu baculis odtodecim \& expanfo tegmine linteo, in fylvis e lignis fimilibus; in Alpibus conftat folo tecto terræ impofito, in Sylvis gaudet parietibus ulnam altis. In centro ejus eft focus, in fronte locus pro culina vacuus, ad latera thalamus communis. Stragula, pelles ramis Betulæ nanz inftratæ. Sic vivit in Sylvis, nec fementem facit, nec metit, eum tamen alit Deus optimus optime.

\section{IV.}

Antediluana Ædiia,

Edificiorum antediluvianorum memoriam nobis confervarunt Sacrx Literx; prima eorum mentio fit Gen. IV:17. Poftea Cain urbem condidit, eamque de nomine flii fui Hanoch appellavit; \& v. 20. Jabal fuit Pater inbabitantium Tentoria, \& pafcentium Gregem; qua Urbs (Gnir) a Caino condita probabiliter fecundum Doctiff. CLER CUM tenuis fuit Cafarum collectio, maceria forte aut fxpe viridi cincta. Nam hac Voce apud Hebraos etiam unum ædificium defignari folet; ita paffim sionis Arx Urbis Davidis nomine nominatur, uti 2. Sam. V:7. Et Jabal dicitur primus eorum, qui Nomadicam fub Tentoriis vitam egerunt, qux ambulatoriæ vitæ Ratio etiam poft Diluvium multarum fuit Gen-

tium

(c) Iter Alp. 1: anni 1702. Lugd. Bat. 1723. 4. p. 54.

(d) LINN IE 1 Hora Lapponica. Amf, 1737, 8, p, 269. 
tium circa Palxttinam Populorum \& ipforum quoque Ifraelita* rum, dum in deferta Arabia degebant, \& Abrahami, Ifaaci, Jaccbi, uti de Abrahamo legimus Gener. XII:\%. Tranffulit autem (a Choreb) ad Sechem, ad montem ab Oriente ipfi Betbel, tetenditque illic Ta. bernaculum fuum : Quod ipfe divus Apoftolus P A ucus laudem fidei Abrahami \& Patriarcharum in Epiftola ad Hebraos Cap. XI. v. 9. commemorat, per fidem Abrahamum vocatum aufcultaffe DEO, ut abiret in locum; quem accepturus erat pro hareditate, \& exiiffe, nefcium, quo effet venturus, per fidem eum moratum effe in Terra Promiflionis, ut aliena, ut qui habitarit in Tabernaculis cum Ifaaco \& Jacobo cohæredibus ejusdem Promilfionis. Atque hæc vivendi Ratio in tentoriis etiam pofterioribus temporibus his ipfis in locis Orientis paffim apud alias Gentes ufitatiffima fuit. De Arabix Populis apud P L I rum (e) legimus, Nomadas inde infeftatoresque Scenitæ claudunt, \& ipfi vagi, fed à Tabernaculis cognominati, qux ciliciis metantur, ubi libuit. In calentibus illis Regionibus aliisque humano cultu deftitutis, populi in familias divif, tamdiu tendunt in loco, ubi funt, quamdiu pabuli copia eft: quo deficiente tentoria refigunt. De Scenitis Mefopotamix, qux ad Meridiem vergit, incolis STRABO L. XVI, eadem tradit, Paftoritiam vitam agentes facile in alia Loca demigrant, ubi palcua deficiunto

Ipfa tamen primorum hominum vivendi Ratio, dum \& agrum colebant, \& vitam paftoritiam agebant præter Cafas \& Tentoria ædificiorum varia genera \& utenfilia \& inftrumenta diverfa polcebat. Stabula pro animalibus, Repofitoria Vidtus, colendi agri Inftrumenta. Plura etiam, cum multiplicata progenies in Societates coibat, cum Itinera inftituebantur, \& lacus \& flumina trajicienda erant; cum ex Venatione Vičtus quærebatur, cum ex depravatione animi vitia; apertæ inimicitix natæ. hoftiles Impetus facti \& repulfi funt.

Et cum juftiflimus injuftitix Vindex fapientiffimus DEUS Arca cor- chica. 
corruptifimorum mortalium interitum ftatueret, Noachicam familiam, cum vario animalium genere mediis in Cataclyfmi undis rervaiurus; Noacho ita pracepit (f): Fac tibi Arcam de Lignis Gopher, in manfiones interftingues Arcam, Gituminabis cam intus E) extus. Et boc modo facies eam, Trecentorum Cubitorum erit Longitudo Arce, quinquaginta Cubitorum Latitudo ejus, छG triginta Cubitorum Altitudo ejus. Feneftram facies ipfi Arce: Gं in c'ubito con. fummabis eam fuperne. Oftium quoque Arce ipjius, in Latere ejus pones: inferiora, fecunda ES tertia facies in ea. Cum ab inftituto meo alienum fit Architedturz navalis fundamenta hic exponere, non longior ero in demonitrando, quam apta, quam fufficiens \& omnimodo propofico Scopo congrua fuerit hxc fabrica, \& quod in hoc fpatio 2041950 pedes cubicos æquante $\&$ in tres contignationes divifo pro hominibus $\&$ animantibus corumque victu $\&$ reliqua fuppellectili conveniens in Arca locus, tutum habitaculum \& promtuarium fuerit, per integrum anni decurfum \& paucos, qui excedunt dies, tempus nimirum, per quod Noachus in Arca commoratus eft; qui enim de hifce plura cognofcere cupiunt, iis Scripta Virorum Cl. Scheuchzerı, Schmidi fuper hoc Argumento abunde fatisfacient. Inquiramus modo in fpeciem Ligni hujus navigii. Lignum, ex quo Arca conftrui debebat, dicitur Gopber, de quo Interpretes valde inter fe diffentiunt, cum hac Vox femel tantum neque extra hunc locum in Scripturis legatur. Apud Interpretes Grxcos redditur $\xi u ́ \lambda \alpha \tau \leqslant \tau \rho \alpha-$ youvce, ligna quadrangula, quadrata : in verfione Vulgata ligna lxvigata. Sunt qui cum Vossio genus omne lignorum refiniferorum intelligunt; ut etiam pro diverfis Arcx partibus varia ligna fuerint adhibita. Pinum alii faciunt cum Muinftero, qux alias in Sacris Erez vel Berofch, vocatur \& in Affyria rarior habetur. Quidam per Laricem interpretantur. Noftra vernacula verfo \& Dana Abietem transfert. Caftalio dubitanter Terebinthum. Cedrus vel Cedrelate Junio \& Tremelio, atque Cbaldæo Interpreti. Doctillimus Bochartus poft FulizRum magna verofimilitudine afferit effe CUPRESSUM Jemper virentem; quod in Affyria 
\& Babylonia fabricaretur Noachi Arca, ubi præcipua materia fuerit, unde fierent Naves, dum alia ligna fabricandis Navibus in terris illis \& tota vicinia parcius inveniantur; in Babylonia enim, ubi prima humani generis fuere fedes, \& poriffimum in Adiabene ingens fuit Cuprefforum copia. Unde Alexander integram Claffem xdificavit tefte STRABONEL. XVI. \& ARR ANO L. VII. tum \& quod Vox Gopber, fono quodammodo accedat ad Coper, Cuper, Cupreffum; quod arbor prxcelfa, redta, xqualis, teres, \& longo caudice. Cupreffi circa Smyrnam tefte HasselQuist ro (g) alciflima \& pyramidum inltar faftigia ad coelum extendunt, præcipuum Natura in his Regionibus Ornamentum. Quod Cupreffus omnium optime putredini \& Cariei refiftat, bonumque odorem fpargat, unde ea, ex qua valva templi Ephefini fiebant, referente THEOPHRAST O L. II. c. 6 . quatuor xtates refervata duravit, quin \& valvæ Templi D. Petri in Roma, ex hoc ligno factr a Temporibus Conftantini $M$. ad Eugenium IV. Papam per 600 paulo minus annos non tantum incorruptx, fed nitentes \& novis perfimiles permanferant. Mortuorum infuper loculi in Egypto, in quibus cadavera condita (Mumias vocant ) recondi folent, multi ex hac materia facti oblervantur. (h) Elt etiam ejus gravitas fpecifica ea, ut facile aquis innatet $\&$ ad aquam in ratione ut 644 ad 1000 . Confentiunt cum Bocharto, Clericus, Scheuchzerus, Celsius atgumentorum pondere permoti.

Ab Architectura antediluviana ea etiam, qux ad Ornamenta \& Oblectamenta pertinebant non plane abfuiffe ex ipfa hominum vita luxuriofa \& ex eo concludere licet, quod (i) Lamechi alter filius Jubal Inventor fuerit tangendx Citharx \& Teftudinis. Et his Infrumentis ipfa cupreffus optime inferviebat, Ufus ejus, etiam hodie elt ad Menfas \& Tabulas, \& alveolos exornandos, necnon ad organorum Muficorum tubos tibias, reliquaque infrumenta. B

Mulica.

(g) HASSELQUIST Reife nach Palærtina. Roftock 1762 p. 320

(h) RAJ. Hift. Plant. T, II, P, I40\%.

(i) Gen IV: 21 . 
Mufica. Nitorem recipit, ideoque ex hac condere opera lautiora confuevere, uti legitur apud Rajum \& Theophraftum locis citatis.

\section{V.}

Architedur Status polt Dilu. vium.

Mox poft Dilluvium Noachi pietas \& gratiffima in DEUM Servatorem fuum benigniflimum eundemque juftiflimum Mens locum dedit Architecturæ Sacræ, xdificavit enim Noachus DEO Altare obtulitque in illo Hypocaufta Gener. VIII : 9. Servatx in diluvio Noachicæ Familiæ xdificiorum materiam fponte obtulerunt, cum ipla ligna cuprellina Arcx, tum aquarum undis fupernatantes Arborum Trunci in Terra fuperficie poft Aquarum receffum relicti. In Tentoriis porro vivere confueverunt, tum \& agriculturam exercere, vineam colere, (ut etiam norint uvarum fuccum in vinum, inebriantem potum parare) paftoritiam vitam agere, ut id conftat ex Genef. IX: 19,20, 26 .

Multiplicato dein genere humano novas fedes quxrere caperunt profecti ex Armenia in Affyriam, in Terra Sinehar, qua Babylonia, Campum invenerunt, quem inhabitarunt Gen. XI : 2 , Loci etiam amœnitate \& commoditate permoti confilium ceperunt de condenda ibi Metropoli, de adificanda Turri, qua famam ad pofteros propagarent, \& circa quam fedes ponerent. Ipfa loci natura offerebat argillofum folum, quod Igne in lateres coctum lapidum loco ferviret \& Bitumen quod Camenti vicem præftaret. Sed DEO fapientiflimo aliter vifum, confudit eorum labia, \& diffenfu inter eos nato per univerfum Orbem difperfi funt, dum Semi polteritas in fuperiori Afia fedes fuas pofuit, Chami partim in Afia, partim in Africa, Japheti in Afia minore \& Europa. Prout id plenitus ex Sacris \& profanis hiltoriis apud BOCHAR TUM demonftratur. Et in Afia quidem \& Africa Architectura magis excoli coepit, fed potius in ingenti Operum mole, quam in commoditate, ornatu, \& venuftate perfectionem quxlitam fuiffe cx portentofis Babyloniorum \& Ægyptiorum moliminibus, ex eorum Pyramidibus, Obelifcis, concludere licet. Ad perfectionem vero xdificiorum hac tria pracipua requiruntur, ut fint commoda, firma, venufta; hoc eft , ut .copo, cui deftinantur, negotiis in is obeundis conveniant, quod ut tuto fiat, firmitas ea fit, qua per fuffi- 
ciens Tempus perfiftant Ut verò placeant ædificia, accedat infuper partium fingularuminter fe \& ad totum proportio, lateratium æqualitas $\&$ ad medias apta ratio, quam Symmetriam \& Eurythmiam vocant. Ineft enim menti noftræ tacita quædam numerandi \& a:fimandi facultas, qux in Rebus fenfibus obviis quantitatum Gradus \& Relationes confufe cognoficit $\&$ in iis declaratur, quæ fimplicioribus numeris exhiberi poffunt, $\&$ inde Mens quafi quandam Pulchritudinis Menfuram ftatuere folet, eam majorem, quo plures diverfarum hujusmodi Rationum confenfus in data re obtinent. Ex hoc Principio Toni vel fimul vel fuccelfive aurem afficientes in Mufica placent, quarum Celeritates vel undulationum aërearum num meri rationem inter fe obtinent, uti $2,3,3: 4,4: 5,5: 6,2: r_{2}$ I: 1 . \& qua ex his componuntur; hoc ipfum quoque eft principium dijudicandi gratiam \& venuftatem Colorum omnemque pulchritudinem Archited onicam, qux id poltulat, ut cum commoditate \& firmitate partes ædificiorum cum accuratione elaboratæ aptas inter fe rationes exhibeant. Et abfolutilimum fanè fummæ perfectionis \& Pulchritudinis exemplar prabet ipfa Humani Corporis forma externa in vario Sexu \& varia hominis ætate, in ea enim fingulæ Partes inter fe \& ad totum perfectifimam Symmetriam atque Eurythmiam obtinent, ut Architectorum Principes etiam inde regulas pulchritudinis \& rationum, qua placere poffint, defumpferint $(\mathrm{k})$. Admirari etiam hic liceat cum Celeb. WOLFIO (I) \& illuftri de $H_{A L L E R}$ perfectiffimam illam externarum partium Symmetriam, qux fummx pulchritudinis ideam excitat, dum hisfóbjectx Partes internx, vifui non fubjectx, vix aliquam Symmetrix Speciem exhibeant, fed eâ modo perfectiflimâ fabricâ aptoque fitu gaudeant, qux Functionibus a fe exercendis omnium optime conveniunt. Videtur, inquit III de HAI LER (m). hoc Deum Ordinis demonftrare, quem ubique fervet, ubi id fieri poteft, 6 nefcio, quid babet fubtilius. Videtur enim ordinem bomini preferce ma-

(k) Vitruv. L.III. c. 1. p.m. 38 .

(1) WOLF bom (sebroud) ber Theilen in ग)eniden, Shieten uno

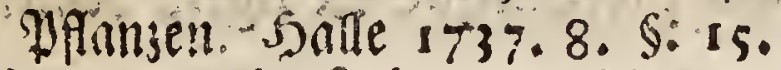

(m) Element, Phyíolog, T, VHI, p, 2, p. 47, 48. Bernx 1766, 4, 
nifeftum, ut manum Creatoris agnofcat; quo vero bomo, nifi Incifor. non penetrat, ea fede, alias quam, Symmetria leges A U C TO REM N AI U R E effe fervatum.

\section{VI.}

Architectura Sacra ejusque praclara fpecimina it Tabernaculo Ifraelitico,

Prima quoque $\&$ antiguillima perfectiflima Architedturæ fpecimina DEUM ipfum omnis Sapientix \& Scientix fontem, Auctorem habent. Maxime illuftre nobis exemplum prabet Tabernaculum in deferto ab Ifraelitis divino juffu juxta prototypum Mofi in monte Sinai oftenfum, confructum, cujus defcriptionem legimus Exod. XXV ad XXXI \& XXXV ad XL. Ad exequendum enim hoc divino cultui confecrandum opus ipfe DEUS, Architedtus fummus, operariis omnibos \& fingulis fcientiam indidit. Locutus eft Jehova ad Mofen ..- Loquere ad filios Ifrael. - Facient mihi Sanctuarium, ut habitem in medio eorum omnium, ut ego oftendam Tibi fimilitudinem habitaculi \& vaforum ejus. - Deftinavi huic rei Befaleél, cui plenam omnis opificii peritiam, intelligentiam, Scientiam divinitus infpiravi, qui negotium ingeniofillime conficiat, aurumque $\&$ argentum $\&$ xs fabrefaciat ; quique gem. mas artificiofe illiget, \& lignum fabricetur. A tque omne artificium exerceat. - Dedi infuper in Corde ejus, ut docere poffit \& alios.-Ei addidi A holibam - inque omnium Solertium animos peritiam immifi, qui omnia quæ Tibi præcepi conficiant : Nempe Tabernaculum Ecclefiæ, Arcam Teltimonii ejusque propitiatorium, \& omnia Tabernaculi vafa, \& menfam \& vafa ejus, purum Candelabrum, utrumque cum omni fuo Inftrumento, Aram Suffitoriam, Aram Victimariam cum omni fuo Inftumento. Labrum cum fuo Scapo, _- Veftes, Operimenta, Aulæa, Atrium \& omnes eorum partes. Et in hoc Templo portatili, præfentis inter eos divinæ Majeftatis certiflimo pignore omnia ad exquifitiflimæ Venuftatis \& Symmetrix leges elaborata fuerunt. Erat arce longitudo $2 \frac{\pi}{2}$ cubitorum, longitudo \& altitudo $I \frac{\pi}{2}$. Menfx longitudo 2 , latitudo 1 , altitudo $\frac{\pi}{2}$. Tabernaculi longitudo interna 30 cubitorum, latitudo \& altitudo 10. Craffities Afferum, ut firmiori bafi inniterentur unius Cubiti. Atrium 100 Cubitos longum, 50 latum, 5 alcum. Scriniaria autem opera plerumque ex ligno scbittim erant 
conftructa. Ex eo enim confecta Arca Teftimonii in inceriori Sancluarii thalamo collocata Exod XXV:Ir. Exhoc Afferes Arcre arrectarii \& Vectes ejus auro obducti Exod. XXV: 13. \& XXVI : 15. Ex eodem Ara fuffitoria Tabernaculi \& Vectes ejus aere ob. ducti Exod. XXVII : 1 -6. Menfa \& ejus Vectes auro obducti. Exod.XXVI:23.26. Thymiaterium fuffitus \& Vectes ejus Exod. XXX:I. ร. Afferes Tabernaculi arrectarii Exod. XXVI: 1 s. item tranitilla afferum ejus. Exod, XXVI:26. (n)

Quodnam vero hoc lignum Schittim Arboris genus defignet, mulum inter fe diffentiunt Interpretes, ut etiam plures verfiones \& noftra quioque Turicenfis hebraicam vocem Schittim retinuerint.

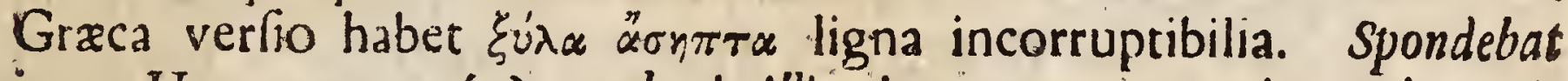
juXta HILLERUM (o) arboris illius incorrupta materia, adorantibus coram Arca, \& in Altari sacrificantibus \& in Tbymiaterio adolentibus DEI Servatoris gratiam. Alii pro Picea, Pinu, Abiete habent, Caftalio Cedrum interpretatur, alii Citrum, Lentifcum, Buxum. Vix autem dubium eft per Sittim Mimosam Niloticam LiN Spec. Plantar. Ed. II. p. I ร06. n. 34. defignari. Eft enim Scbitta \& Scbittim Hebrxorum, Sant vel Sont Arabum (p), Acacia vera, C. Bauh. ipfa Planta a Li N Æo indicata, Spina Ægyptia, "A ravbos THEOPHRASTI (q) cui tribuit materiam duodenorum cubitorum tectis idoneam; qua etiam in navium fabrica ad coftas, ventrisque compagines utantur. Spina dicitur, quod Spinis horreat. Et forte eft hæc ipfa Spinæ albx fimilitudine Arbor, quam H 1 ER o. Nymus ad Joelis Cap. 3.v. 18. ubi de Nahal Sittim agit, defcribit ( $\mathrm{r}$ ). Efi genus arboris in Eremo pine alba fimilis - tam grande, ut latifima ex is tabulata cadantur: lignumque fortifimum eft, in$\mathrm{B}_{3}$ credic.

(n) Conf. Cel. Scheuchzeri Phyf. Sacr. T. I. Aug. Vindel.173 f. p. 197-272. Tab. CLXXIII - CCXXX. Schmids biblis fider Mathematicus, 3ínidan 1736. 8. p. 301.

(o) Hierophyt. 1. 428.

(p) CELs. de plantis facris. 1, 498.

(q) Hift. Plant. L. IV.c. 3.

(r) D. H. URsiNi Arboretum Biblic, Norimb, 1699, 8. p. 314. ex Hieronymo, 
credibilis lavitatis \& pulcbritudinis, ita ut ex bis etiam vafa Torcularium faciant: Addit: Nusquam nif in Arabix folitudine reperiri. Ex ea naves Egyptiorum onerarias factas fuiffe auctor eft H в R ODotus L. II. C. 96. Certe hanc Arborem Schittim copiofe nafci in Arabix defertis ipfe facræ literæ docent apud Efajam Cap. 4I.

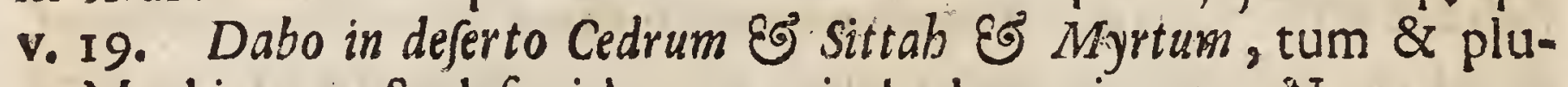
ra Moabitarum \& deferti loca qux inde denominantur Num. $25:$ r. 33:49. Jof. 2: r. Illam verò Acaciam veram S. Mimofam Niloticam, sant Arabum his in locis copiofe inveniri Hodoporicorum teftimoniis indubitatis confirmatur. Dodtifimus (s) BELL ov r us hæc de hac ftirpe habet. Ad littus Arabici Sinus prope Sues Itinere facto per fterilem folitudinem, ubi nullæ firpes nafcebantur, prater Acaciam, ejus Gummi ab Arabibus collectum in Europam copiofe transfertur. Incolæ ejus ramos largis perticis flagellant, ut folia decutiant à pecore devoranda. Et apud P. A LP IN U M de Plantis Egypti ( $t$ ). Acacia, quas Sont appellant copiofifime in Monte Sinai proveniunt ad Mori magnitudinem excrefcentes. Eruditiflimus Celsi us loco cit. teftimonium P. R o ger excitat, quod ita fonat. Le Setbim ne Se trouve que dans l'Arabie deferte, procbe de la Terre des Madianites, peu éloignédu Mont Sinai, en un lieu, qu'on appelle Setbim. - C'eft Arbre eft auffi grande comme un Noyer, fon bois eft leger, de très bonne odeur EG incorruptible. Unicam fere effe arborem Arabix petræx, qux conficiendis afferibus fervire poffit ex Rauvvolfii Obfervationibus affert Gronovius (u) \& confirmatur a Doetiff, $S_{\text {H }}$ vv (vv) in fubjuncło Itineri Orientali plantarum Catalogo. Infigne quoque his Doctifimorum Virorum BochartI, Salmasit, HiLleri, SCheuchzeri, C'elsit, aliorum placitis, quibus Schittim ipfa hac Acacia eft, pondus addit accuratior hujus plantx defcriptio ab accuratillimo Botanico

(s) Obfervo Lo II. c. s.

(t) Alpini de Plantis Egypti liber c $c_{\text {i }}$ Obferv, \& Notis. Jo. Veslingii. Patav. 1640, 84, p. 9.: \& Veslo Obferv, p. 8.

(u) Flor. Orient. $\varsigma_{0} 159$.

(w) Voyages dans plafeutrs Provinces de Ia Barbarie \& du Levant, à la Haye 5743 , 4. T. II, po IOI. n.' 3 . 
HasSEL QUistro (x) in Oriente confignata. Eft Mimofa Nilo. tica in Egypto inferior rarior \& in hortis plerumque culta : nafcitur autem fponte in deferto arenofo prope antiqua fepulchra $\mathbb{E}$ gyptiorum in Ægypto fuperiore ubi integræ ejus Sylvæ ; itemque in Arabia petræa, ubi Gummi Arabicum ex ea colligitur magna copia ad Sinum borealem maris rubri ad Montis Sinai pedem, ubi Gummi Thuris inde provenit. Arbor eft ingens, ramofiffima, gummora, cortice purpurafcente, floribus capitatis luteis pedunculatis, leguminibus compreflis articulatis, moniliformibus, Spinz binx longiores, quam in Acacia Farnefiana, diftinctz, ftipulares, folia bipinnata, partialibus extimis glandula interftin. ctis, Icon habetur apud BELLON. I. c. VESLINGIUM de plantis Egypt. T. VIII. Pluknetum Tab. I23. f. r. Confundi folet cum Senegalenfi \& Farnefiana. Senegalenfi funt Spinæ ternx, flores fpicati, legumen compreffum, lave, ellipticum. Cortex albus. Etiam hanc in Arabia provenire afferit Cel, Li N N \& us l,ce.

\section{VII.}

Alterum Architectüræx Sacre abfolutiffimum Specimen prz- In Temp bet Templum Hierofolymitanum primum illud in Monte Moria a Salomonis S A LOMONE (y) Anno Mundi 2932 inchoatum \& feptem annorum fpatio abfolutum fuit, a Nebucadnezare vero anno 3360 deftructum $(\mathrm{z})$. Tum \& alterum vel reftauratum Templum fub Zorobabele (a) Anno 3485. quod amplificatum \& ornatum fuit ab Herode Magno, funditus dein everfum a Romanis Anno poft Chriftim natum feptuagefimo. Erant in hoc Templo Salomonæo Dei omnipotentis æde, quam ipfa deinceps JeH OV Æ gloria impleverat (2. Chron. $5: 24$.) omnia ad magnificentiam, perfectionemque maximam comparata five Fundatorem (pectes, five Directorem primarium \& fecundarios, five Operarios numerofif-

fimos,

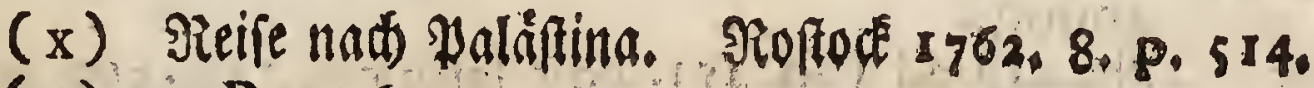
(y) i. Reg. $6: I_{\text {. }}$
(z) 2. Reg. $25: 9$.
(a) Esdr, Cap, 3. 
fimos, five materiam xdificii \& partium ejus, five adificium ipfum cum paribus fuis, five contenta ejus, \& vala, Inftrumenta, atque univerfum apparatum; prout luculenter \& graphice hac cuncts in facris literis r. Reg, 6. \& Chronic. Cap. 3 \& 4. defcripta exhibentur \& Dodtiffmorum Virorum VILALPand (b) pracipue, \& L. Chr. Sturmia (c) Scheuchzeri noltri (d) \& SCHMIDII (e) Commentariis illuftrantur. In quo argumento eas potiffimum Templi partes confiderabo, qux ex ligno fuerunt confructe, quas ita expendam, ut nexus quem cum cxteris

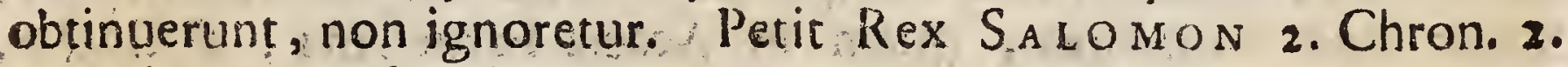
v. 8. à Hiramo fibi de Libano mitti ligna Arafim, Cedrina, Berofcbim, Algumim pinea, picea, ad Templum ingens \& admirabile, domum nomini JEHOV E DEI contruendum. Hujus ideam a DA VIDE acceperat; ad quem omnia venerunt fcripta manu Domini, ut intelligeret univerfa opera exemplaris (1. Chron. 28. v. 19.). Dedit namque David Salomoni filio fuo fimilitudinem porticus 6 domuncularum, E cellariorum, E conaculorum, \& cubiculorum ejus interiorum, domusque propitiatorii, tum exemplar omnium eorum, qua babebat in animo \&o omnium cubiculorum per circuitum in thefauros Dei, GG in tbefauros Sanctitatum . tum ad Sacerdotum Levitarumque claffes pro cuncto opere Minifterii nempe pro univerfis vafis minifterii domus Dei. Domus fancta five Templum proprie dictum quod Sanctum \& Sanctum Sactorum complectitur, longum erat 60 cubitos;20 latum, 30 altum. Ante Templum Veftibulum, Pronaon, 10 cubitos latum, 20 longum, cujus frons 120 cubitos alta (2. Chron. 3. v. 4. I5.16, 17, ante hoc dux columnx 2 nex 35. cubitos altz: qua faciei Templi Orientali prapofitx erant. A Veftibulo Sanctum Muro erat diftinctum, in quo Porta magna 20 cubitorum alta, Io lata gémina, cum duabus valvis è lignis

pineis

(b) Explicationes in Ezechielem. Romx 1604. III. Vol. f.'

(c) Sciagraphia Templi.Hierofolymitani. Lipr. Ir94, 4, \& in notis ad Goldmannum.

(d) Phyl Sacr. Tabo 49 ad Tab 4530 edit latina p $519-566$ edit. germanica T. II. p. $121-180$

(e) Bibl. Mathemat. p. 317. Tab.15-17. 
pineis (Scbernen). Sancti longitndo dupla longitudinis Sanctifimi 40 fcil. cubitorum, latitudo xqualis alticudini, cubitorum 20. Intrinfecus verfus Occafum erat Oraculum, sandum Sanctorum, Sanctitas Sanćtitatum figurx cubicæ 20. Cubitos longum, latum, altum. "Huic \& Sandto impofitum laquear convexum ex Lignis Cedrinis, to cubitos altum, quod altitudinem à vertice ad bafin efficiebat 30. cubitorum. (I. Reg. 6:2,) Sanćtiffimi oftio velum prxtendebatur pretiofilimum ex Hyacintho Purpura, Cocco \& Byffo c. Cherubinis (2. Chron 3: 14.) Oftii valvæ pinex auro obductz, fuperliminare \& poftes quinque laterx. Templi parietes ex marmore candido faxis arctiflime compaginatis tabulis cedrinis (Arafin) obducti, pavimentum abiegnis (Berofcbin). Sed hoc ipfum pavimentum \& parietes interni totius æxdis \& januz auro contecta \& \{culpturis extantibus Cherubim, Palmarum atque florum exornata. In Sanctiffímo bafi marmorex impolita fervabatur Arca foederis, qux in Tabernaculi penetrali fuit ex ligno Sittim conftructa, \& auro obducta. (v. 20.) Arcx adftabant duo Cherubini figura Coloffea 10. cubitorum, quorum alx Aram ejusque Veftes operiebant. In hoc Sanctuarium Soli Sacerdoti fummo aditus femel in Anno concedebatur. In Sancto vero fervabantur Arca fuffitus cedrina, menfa panis propitiationis, candelabra aurea $(v, 20)$ Addita verò Sancto \& Sanctiffimo Conclavia plura fuperiora, itemque lateralia , tum duplex circuitus triplicis contignationis $(v, 5.6$. ) fuis gazophylaciis inftructus : \& deinde atrium duplex interius Sacerdotum, atque exterius \& magnum. (2. Chron. 4:9.) Populi Ifraelitici.

Hxc autem compendiaria Templi Salomonis delineatio ex facro codice deprompta, ex eodem egregie illuftrabitur, fi conferantur cum defcriptione Templi EzECHIELı Prophetæ (f) à fummo DEO in Vifionibus A. M. 3375. reprxfentati, ex quo Judxi Jacturam quam pafli fuerunt agnofcerent $\mid \&$ reftaurandi fub Zorobabele Templi pleniorem ideam haberent, fimul ut hac adumbra-

tione 
tione Templi Myftici Novi Teftamenti viva in fidelibus (pes divinx falutiferx gratix, \& gloriofiffimi Ecclefix tzatus, DEI in Sanctiffimo Templo ad fuum Populum in fempiternum habitan: tis efficacilfime excitaretur. Ex geminis verò Templi Salomonei \& Ezechielis defcriptionibus collatis cum iis, qux de Templo Hierofolymitano apud Jofephum atque Judxorum Scriptores proftant, natæ funt defcriptiones \& delineationes, quas in Auctoribus fupra laudatis invenimus, qux fplendidifimi operis pleniorem ideam exhibere poterunt: quamvis non difficendum fit, in his ipfis ingenium Architectorum varia, in defcriptionis fupplementum addidiffe. Taceo duo alia Templa ad Hierofolymitani fimilitudinem exftructa; Samaritanorum in Monte Garizim ad Samaballete \& Heliopolitanum ab Onia IV. fub Ptolomæo Philometore \& Cleopatra in Egypti prafectura Heliopolitana. De praftantia \& excellentia Architecturx hujus Temporis judicium ferre licet ex Magnificis Ædificiis \& Palatiis Regiis à Salomone ad exquifitiffimas Symetrix leges exftructis. Domum Cedrinam David inhabitavit (I. Paralip. 14:1. \& 17: 1.) ad cujus conftructionem Hiramus Tyriorum Rex materiam cedrinam \& Lignärios fabros miferat. Regia \& fplendiffima Salomonis Ædificia (g) (I Reg. Cap. 7.) fuere Regia Salomonis Domus cum atrio, opus 13. annorum, Domus faltus Libani Palatium 100 cubitos longum, so latum, 30 altum, quatuor columnarum cedrinarum columnis inftructum, quibus cedrina tranftra incumbebant, cujus tabulata cedrina fuper 45 columnas, Luminum triplex ordo, feneftris rectangulis triplici verfu \& ferie inter fe refpondentibus; Columnarum porticus so cubitorum longitudinis; Porticus Solii atque Domus Pharaonis filiz, quam duxerat. Erant quoque columnæ tum Templi, tum Palatiorum Regiorum in Capitulo, in Trabeatione, in Zophoro omni genere ornatus Archirectonici Liliorum petalis (Scboschin), Granatorum fructibus (Rimmonin) reticulatis operibus pulcherrime exornatx (I.Reg. $7: 18-24$. 


\section{VIII.}

Lignorum diverfa genera qux in memoratis Ædificiis impri- Lignor $m$ mis in Templo adhibita fuerunt requentia denominantur Arafin, genera Berofchin, Schemen, Algummina, Almygim, qux ut plurimum per ficando Cedrina, Pinea, Oleaginea, Ebena aut Brafilia reddi folent.

De Lignis Arafin \& Berofcbin in fuperioris Differtationis Phytographix Sacrx parte V. J. 9. fulius egi. Probabile nimirum videtur, Vocem Arafin generali nomine defignare Arbores, refiniferas quafcunque coniferas zque ac bacciferas PıNos \& in his Abietes, Cedros, Larices. Cupressos, Thuyam, JuNIPEROS earumque Species fabinam, \& Cedrum phæniceam, alias : Speciatim verò Cedrum Libani per emphafin denotare præftantiffimam illam $P_{1}$ I fpeciem qux propriè Cedri nomine venit, \& gratia odoris, incorruptibilitate, denfitate \& prxftantia Ligni præ cæateris expetita pretiofioribus fcriniariis operibus tabulis, arculis, vafis adhiberi folet. Neque ideo negamus JUNIPERUM. phæniceam, quæ fubinde Cedri nomine infignitur, ejus loco infervire potuiffe. Majorum verò operum Trabeationum, Columnarum multò maximam partem ligna Pinea \& Abiegna fuppeditaffe, qua promifcue nomine lignorum Arafin defignare folent, nifi fignificanter per Berofch aut Beroth Pinus indicetur, cujus Ixtum in his Terris proventum fuiffe fupra vidimus. Verfio græca

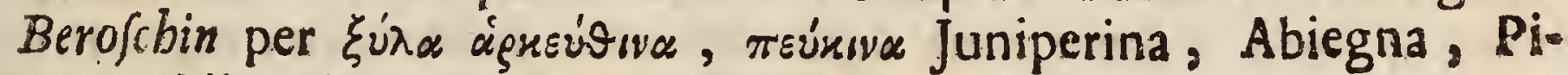
nea reddit. Plura videas apud Celsium, (h)

Ex Ligno Scbemen Cherubinos ( I. Reg. 6. v. 23, ) valvas oftii Oraculi (v:3r.) \& poftes oftii Templi (v. 33.) fabrefactos fuiffe legimus. Vox hebraica proprie defignat ligna pinguia, quam alii per oleaginea reddunt, alii per Olex ligna; Græci Interpretes promifcue vertunt Cypariffina, Arceuthina, Peucina. Turicenfis verfio latina ligna pinea, vernacula ligna Olex interpretatur. Cum autem Olex apud Hebrxos in Sacris proprium no- 
men, Sait, darum lit, a lenlu $S, S$ non aberrabunt, qui pro lignis scbemen hebent omnis generis ligna, qux ob oleofarum partium abundanciam, ob texturz denfratem, \& fibrarum teneritudinem, poliuram, \& nitorem admitunt, qui operibus ligneis venuftarem \& folendorem conciliare pollic. Huic fopo imprimis conveniunt Olea, Juglans, Juniperus phanicea.

De Lignis Alguminin legimus, fuiffe inter ea qua de Monte Libano fibi mitti Salomon petierat 2. Paral. 2:8. \& Cap 9. v. 10. Ir. Servos Regum Hirami \& Salomonis cum Auro ex Ophir attulıfe quoque ligna Algummin, non ante vifa in Judxa. Et 1. Reg. To. v.-11.\& I2. Claffem Hirami ex Ophir quoque attuliffe magnam valde copiam lignorum Almygim, \& ex his Salomonem feciffe fulcra, Citharas \& Lyras nec vel antea vel poftea hujusmodi ligna vifa fuiffe. Ex quibus inferre licet Ligna Algum. min \& Almygim non differre, fed voces per metathefin literarum mutatas idem defignare lignum; deinde ligni hujus aliam fpeciem ex Monte Libano, aliam ex Oriente fuif́te petitam. Non in alia voce interpretanda Auctores magis inter fe differunt, quam in hac. Verfio noftra latina habet Corallia, qua voce Hebræi Litophytum illud rubrum, quod Corallium rubrum vulgo dicitur, LINNAO (Sylt. Nat. I. p. 797 -38.) MADREPOR A rubra denotant. Hoc vero operibus, quibus à Salomone adhibitum fuit, minime conveniebat. Vernacula Verfio reddit Lignum Ebenum; Ebenbolz, quo nomine varia ligna denfiora, obfcurioris, nigricantis coloris, polituram admittentia defignari folent \& fpeciatim EB E u U Cretica Lin n. 1076. Atbiopica quæ Palmæ fpecies Linn. mat. m. 504. Indica vel viridis Inf. Helenæ. D A I E mat, m. p. 330. Jamaicenfis Slo a n.e Hift. II. 30 qux ASPALATHus Ebemus LIN N. n. II. His annumerari poffunt variæ Lignorum Species ex Claffe Arborum Diadelphiarum, \& Leguminofarum Cytifi, Robinix, Mimolx, Cafliarum, quorum ufus permifcuè in officinis fabrorum lignarıorum aliorumque mechanicorum in vicem Ligni Ebeni habetur. Hiclerus (i) \& alii ex rationibus Etymolo- 
gicis permoti hoc nomine omne genus arborum gummiferarum gummi aut refinas ftillantium defignari exiftimant: \& harum Arbo. rum tum Montis Libani tum Indix Orientalis Catalogum dedit HiLERUS 1. C. PAGNanUS \& alii rediunt Ligna Brafilia rubra; Sed Hirami Temporibus in Indiam Occidentalem navigatum fuife non conflat. Verfo grxea haber ligna peucina, dedolata. Verfio vulgata Thyina. Alii \& in his CELsıus (k) ligna pretiofa ex India Orientali, an Sandali Lignum? Verofimile videtur Algummim Montis Libani fpeciem fuiffe ligni praftantioris ; durioris, quod polituram admittere \& pretiofis operibus rervire poffet, cujusmodi Ebenus \& varia ligna floribus papilionaceis \& in his fpeciatim SHARTIUM Spinofim LINN. 997. quod in Syria \& Grxcia copiole crefcit in Sepibus \& Sylvis. Ligni materies alba durillima \& gravis, medium autem Ligni nigrefcic. (1) Notum etiam eft, hifce Lignis majorem nigredinis gradum conciliari, dum atris pigmentis immiffa atrum abforbent. Ligna verò Algummim ex Opbir ad lata fpeciatim defignare videntur præftantillima. Indıæ Ortentalis ligna, qux in pretiofis operibus \& ob gratiam Coloris \& teneritu. dinem fibrarum atqua denfitatem texturæ cxteris præitabant, præprimis Santalum rubrum, quod hoc ipfum Corallia rubra, qux alias per Algummim denotari folent, fuo colore quodammodo referat; fed \& S ANTAL UM citrinum \& album, quod colore, teneritudine Ligni, \& imprimis gratia fuaviltimi odoris placere \& in pretiofis operibns adhiberi poffit. Nafcitur in Indiz Orientalis variis locis imprimis in Infula Timor, unde in Sumatram transferri folet, Infulam Auri feracem, quæ à nonnullis Ophir Veterum creditur; dum alii Infulam Ceylan Ophir Arabum fuiffe exiftimant. Id certò conftat frequens utriusque loci effe commercium, ut omne Ge. nus Mercium ex Summatra ad Infulam Ceylan deferri potuerit. Eriam in Infula Timor Santalum rubrum nafci ex J EEGERO RuMPHI US ( $n$ ) tradit, cui triplex Species SANTALI rubri I. C 3

ex

(k) De Plantis Sacris 1. 171. \& U U U1N. Arboret. p. 565.

(1) Jo. Bauhini Hift. Plant. P. I. L. XI p. 376.

(im) Garcin apud Savary Dictionnaire de Commerce. à Geneve 1750. f. Pref. 34. Tom. 11 . p. 762,

(n) Rumphis Herbar, Amboin, Amltel, 174I, f, L, II, p. $42-83$, 
ex Ora Septentrionali Coromandelienfi, quod dicitur Lignum $\mathrm{Ca}$ liatour. 2. Malaicenfe. 3. Timorense, quorum omnium infignis uiu fit in Arte Tinctoria, ad omne genus fuppellectilis, qux per Scri niarios aut Tornatores elaborari folet. Indi etiam Idola \& delu bra fua ex hoc ligno parant. Caterum iisdem ufibus fervire po terunt varia ligna rubro colore prodita \& polituram admittentia quorum ufus in Arte tinctoria imprimis celebratur, ut Lignur rubrum Braflianum five Fernambucenfe. Hxmatoxylon Campecbio num, Cxfalpinia Brafilienfis \& Veficaria \& quæLignum Sappan fup peditat.

\section{$\mathrm{XI}$.}

Vegetabilium ufus in vario Edificiorum genere prabent, in fuppellectile varia dom architedu fica, in Utenfilibus, Inftrumentis, Machinis Oeconomix RL nomiaRufti-fticæ, Urbanæ, privatæ, \& publicæ facile convincimur innumerab ca \& Urba- les effe eorum ufus, Fiunt ex his \& lignis potiflimum Ruricol rum $\mathbb{E}$ dificia pleraque fimpliciores Cafr, ampliores Ėdes Patri familias, Agricolarum, Villicorum, Operariorum: Reconditor Frugum, Horrea, Area, Falifca; Promptuaria efculentorum, pc tulentorum, cellaria omnis generis; Hydrophylacia, alvei, tul 8. vafa aquam fontanam ducentia, effundentia, excipientia. An malium variorum fabula Jumen:orum, Equorum, \& varii gen ris Quadrupedium; Aviaria volatilium, Pícinæ pif́cium, Cancro rum, Cochlearum Receptacula. Suppelledtilis Ruftica omne g nus, Agricolendi Inftrumenta, Aratrum cum fuis partibus ad ve tendam, profcindendam, lirandam Terram; itemque Crates, $P$ ctines, Volvuli ad Terram vel apte mifcendam vel æquandam: Fu carum plures f́pecies, manubria \& apparatus Intrumentorum quorum ufus eft in fodienda Terra, demetendis frugibus, fecanc fono. cædendis lignis, cæteris. Pro frugibus depurandis Cribr Incernicula, Vanni, Ventilabra. Molendinarum varia genera Molx verfatiles pro terendis fructibus, Torcularia \& prela pro Uvi rum, Pomorum, variorum frukluum mufto aut oleo Fructuun nucleorum, feminum. Provehendis \& deportandis oneribus veh cula varia, plauftra, Trahx, \& Jumentorum officiis congrua $j$ 
ga, Sellæx, Clitellæ, funes varii. Solidorum \& liquidòrum menfura cava. Vara ex Gratitio Opere, Corbes, Fifcellx ex Scirpo, Junco, Salice, viminibus. Et huc pertinet cifta juncea bitumine \& pice oblita, in qua Mofes Nilo flumine exponebatur (Exod II: 3 ) contexta ex Game CyPER o Papyrus didto \& repofita in suph Fu. co barbato \& rubente, aliis Alga vocatur. His accedunt varia vara lignea pro affervandis liquoribus vel ex Lignorum compaginibus confructi Cadi \&. Doliorum variæ fpecies, itemque lagena, quales etiam à Lapponibus (o) ex Corticibus Betulx confrui fo. lent, vel quas Natura ultro offert in fructibus variis Cucurart lagenaris, verrucofa, Melopeponis, Americane oblonge bicubitalis. CRESCENTI 2 Cujete feu Cucurbitiferæ arboris, ex cujus fructus Cortice, Ollx, Corbes, Lances, Cochlearia, Ligulx defpumatorix, variaque fuppellex apud Americanos paratur. $(p)$ His adde vincula varia ex lignis, ramis, Corticibus, viminibus, Stolonibus, culmis tenacibus pro firmandis variis ex ligno compaginandis vafis, doliis, aut colligandis fafciculis, aut contexenda varia fuppellectile. Huc porro referenda varia fulcra, funtentacula, perticx, fcipiones. Suppellex domeftica, Menfx, Scamra, Ciftr, Arculx, Patina:, Orbes, Cochlearia, Januarum repagula, Clauftra ligadhibentur Herbx afperæ, Equifeta, Cortices fuberini, alia. Lucernx, faces ex lignis refinolis pinguibus vel eorum radicibus, ut PI I Sylveftris, Elychneia, Oleum Lucernarum, Fomites pro excipiendis Ignis fcintillis : \& quæ alia plurima his adnumerari porfunt, quorum notitia ex Scriptoribus Rerum Rulticarum peten-

Deinde in Architectura \& Oeconomia Urbana non minor Vegetabilium utilitas patet; cum privatim aut publice plurima depro.

mun-

(o) Linn. Flor. Suec, Edit. II. \$. 859.

(p) Jaceuin. Seled. Stirp. Americ. Hiftoria Vindob. 1763. fol.

(q) Mr. de Condamine Mem. de l'Academie Royale des Sciences. 1732. p. 308. T. XVII.

(r) Scriptores Rei Rulticæx cur. J. M. Gesnero Lipf. I735 4to. Car. Aeuino Nomenclator Agriculturæ, Romæ 1736. 4. 
muntur, ad 价ficia, ad Machinas, ad Inftrumenta, ad fuppellectilem domeficam, ad varias commoditates \& oblectamenta. Ex Lignorum Truncis, pro Ædificiis Tigna, Caniheria, Tranftra, Columnx, Afferes, Tabulx, qux ferviant fubftructionibus pro palis in firmando, limofo \& paluftri folo, vel pro tignis palorum capita copulantibus, vel pro cratibus arenaceo vellimofo folo imponendis, vel Liminibus, poltibus, columnis, pilis, paraftatis, aut Tabulatis verticalıbus, horizontalibus, Januis, Feneftrarum calathris, Scalarum gradibus, Tectorum fulcris, deliquiis. Ex lisdem parantur pontes, quibus Vallibus disjuncta loco junguntur, aut fluminum ripa. Et huc referendi varii pontes penfiles ex caulibus Herbarum Sarmentacearum, Lianes, Paulinia, Calami, aliarum, quarum tenaciffimi caules ad 200 vel 300 pedum longitudinem exporriguntur mirificx inter fe implexi. (s) Ex Arboribus $\mathrm{Na}$ vigiorum omne genus five fint Trabes vel Afferes fimplices aqua innatantes, five inter fe in Crates colligatx, five naves monoxylz ex cavato ligneo Trunco Alni, Betulx, Sloanex, Bombacis Ceiba, Caftanex malabaricx, aliis paratx, five Lintres, Scaphx, Carinx, Galeones, naves mercatorix, onerarix, bellicæ, ingentes illæ navales machinx, qux pro Hominibus, Animalibus, Vistu, Potu, Mercibus, Machinis bellicis locum fuppeditant : earum præterea partes \& fuppellex gubernacula, brachia, remi, mali, \& qux ex Vegetabilium Corticibus \& caulibus parantur vela \& funes.

Deinde in Architectura Hydraulica in conftruendis Aggeribus, qui fluminum aquas coerceant \& curfum dirigant, in parandis aqua: rum clauftris, aquaductibus, tubis, alveis, pro aquarum colleetione, diftributione, derivatione, in machinis variis \& Antlis, quibus aqux atcolluntur.

In Mechanicis Operibus plerisque five fuerint fimpliciores Machinx, Vectes, Trochlex, plana inclinata, Cochlex; aut compofitarum mille genus, inprimis tot molarum genera, qux vel terendo, tundendo comminuunt, aut comprimunt, aut denfant, aut ferra fecant.

(s) Adanson Familles de Plantes, à Paris 1.763, 8. pag. $x_{0}$ Pref. CCVIII, 
In Architectura Militari ex iisdem Aggeres \& Munimenta (conf. Deuter. $X X$ : 20.) Cortina, machinarum bellicarum fulcra \& fuggeftus fiunt \& pracipua materia pyrii pulveris, Carbones.

Vegetabilium non minor eft ufus in Venationibus, in jaculis, Baliftis, in plerisque machinis \& retibus, quibus Animalibus infidix parantur. Corylus dat virgas pifcatorias, Vifcum gluten, quo invifcantur aves, Suber \& Cortices populi levem fubrtantiam, qua retia in aquis fufpenduntur.

Ex Vegetabilibus innumerx partes fuppellectilis bene inftructæ domus, quam Scriniarii, Tornatores, Sculptores parant ad inftruendam vel ornandam domum. Tabulata, Repolitoria, Cifta, Arculx, Vafcula, Menfx, Sedilia, Scamna, Vafa, Dolia, Infundibula, Cola, Cribra , Folles, Scipiones, ex variis lignis vel fitipitibus vel calamis arundinun farctarum, vel Radice AMOMI Zingiberis in rectitudinem pondere extenfa. (t) Et ligna tandem, mortuis, Sepulchralis Cift materiem dant.

Ex iisdem tot Machinx pro omni genere Artificiorum itemque materies, quam Artifices in Officinis \& Opificiis ad varios ufus parant \& elaborant. In manuariis operibus non modo plurima ope Lignorum \& Carbonum igne elaborantur fed Vegetabilia ipfam materiam fuppeditant Tinctoribus ad tingendum, Coriariis ad denfandum Corium, Typographis Oleum \& Fuliginis atrum pigmentum ; \& Chartarum omnem materiam, fi Sericum excipias \& Lanam. Neque vero ex Papyro Cyperi fpecie, ex Moro papyracea ex Cannabinis variis plantis, ex linteis, fed \& ex plurimarum Plantarum fibris parabilem effe, experimenta plurima docuere, (u) \& inpr. nupera Viri PI. Reverendi \& Celeb. D. S C H АFFER (vv)

(t) Rumphn Herb. Amb. L. VI.

(u) L'Art de faure dí Papier, par Mr. de la Lande. Paris i $761 . f_{\text {. }}$

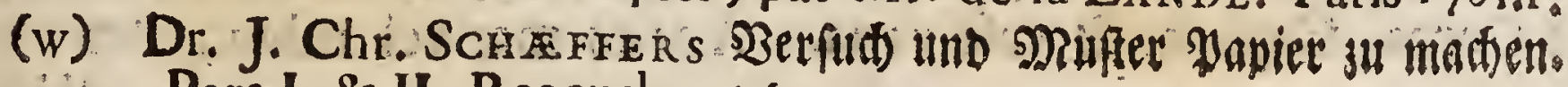
Pars I. \& II. Regensb. 1764, 4. 
in chartaceis fpeciminibus, de hoc argumento editis, in quibus charta varia ex Pappo Populi \& Salicis, ex Ligno Fagino, Salicino, ex Clematitide, ex Lupulo, ex Mufcis, ex Scobe, ex Bracteis lignorum dolabrâ refecttis parata confpicitur. Etiam Sal, quod in conficiendis Vitris Silici admifcetur, ab herba Kali Alcali dictum ex Salfola, Salicomia variisque Sale abundantibus inprimis herbis maritimis paratur.

\section{$x$.}

Varietas \& zopia Negeabilium, qua in A ro hitectura ¿ Oecono. nia varia dhibentur.

Sed in ea rerum utilifimarum ex Vegetabili Regno depromptarum ubertate, quam nemo aut fermone, aut cogitatione facile affequi poffit admirari imprimis licet eam divinam fapientiam omnipotentiam, benignitatem qux tot Vegetabilibus tam diverfas dotes impertivit, \& immenfam eorum varietatem \& multitudinem conftituit, us tot diverfis ufibus adhiberi poffent atque innumerabilibus finibus aptiflime refponderent. Ita Arborum variarum varium eft ingenium, dum harum aliz proceritate rectitudine Trunci, alia craffitie, alix robore, alix Ligni tenacitate mollitie, denfitate, raritate imprimis gaudent, alix in humido, alix in ficco magis perdurant, ut Architectis \& Opificibus optio detur Édificio cuique ejusque partibus maxime congruam materiam feligendi. Ipfa fane Helvetia noltra hon exiguum Arborum numerum fuppeditat, qux ad Edificiorum firmitatem \& omatum, atque ad omne genus fuppellectilis omnem materiam \& praftantiffimam eam prabere poffint. In his funt Quercus Robur, Laudatur ligno duro, tenaci, fubflexili, validiflime in fitu perpendiculari refiftente, longxvi, fi ab acceffu aëris humidi impediatur ; fub aquis in lapideam duritiem indurefcente; hinc ejus ufus in fubftructionibus, in poftibus \& fulcris, Doliis vinariis, in carinis Navium majorum, in prelis, radiis rotarum, Inftrumentis variis : Cortex in Scobem comminutus Coriariis pro denfando corio fervit; Gallx ad pannos atro colore inficiendos \& ad atramentum. A B IES quX PINus picea LINN:Ob levitatem, altitudinem, rectitudinem, refiftentiam, in contignationibus in primis laudatur, ad afferes \& Tabulas, ad malos Navium; ad Cupas, Gapfulas \& vafa varia. A bies mas vel rubra, PI Nus 
Abies L I N N materies Ligni facilior fabris, in opere deterior, pro aëris conftitutione crefcit vel detumefcit, fubinde crepitat, PIN fylveftris Lignum conftans, leve, fiffile ad afferes, fcandalas \& varia utenfilia itemque ad malos Navium fervit. Pinus Cembro iifdem ufibus fervit. P INus Mugho impr. in humidis laudatur \& ad vafa Operum Lactariorum; P In us Larix prx cxteris laudatur, intra aquas perennis, à carie immunis, flammam difficulter concipiens, fed conceptam alens pertinaciter: Feneftrarum Crucibus, poltibus, afferibus, fcandalis inferviens. Hæc verò ligna refinofa à locis igni propinquioribus ftudiofe removenda. JU NIPERUS communis ob fuaveolentiam commendatur \& durabilitatem; ad palos \& fepimenta in Suecia adhibetur; quæ inde parantur pocula medicata habentur uti \& Hederz. T $T_{A}$ Xus baccata Lignum inter Europxa praftantiflimum ruffum, venofum, durum, glaberrimum ad fubtiliora opera aptiffimum ad Epiftomia, Cantharos, Cochlearia : ad Inftrumenta Múfica, ad Dentes Rotarum ad vehiculorum \& Ro: tarum axes, ad poftes. Buxus Lignum habet durum, tenerum quod polituram admittat, manubriis, capfis varisque elegantioribus operibus qux Torno elaborari folent aptum: duritie \& amarore Vermibus refiftit. JUGLANS regia menfis, utenfilibus, operi tectorio, lectis, fcriniis elegantioribusque operibus convenit, \& polituram cum tela cerea admittit. FA G us, ligno eft duro fub dio facile, fub aqua fero putrefcente, aqua maceratum Vermibus magis refiftit, ad Inftrumenta Agriculturæ, Aratra, Plauftra, ad carinas $\mathrm{Na}$ vium adhibetur, in tenues laminas fiffile pro capfis, vaginis, librorum tegumentis fervit. CASTANEA Trabes, Tigna, Scriniaria Opera, Cadorum coftas fuppeditat. CARP IN us ligno gaudet tenacifímo, immenfa pondera fúftinet, ad Trochleas, Mo: lendinarum rotas, tudicula, molarum piftilla fervit. Poputus tremula lignum habet levifimum, candidifitmum Tornatoribus expetitum, inde Clitellx, Calopodia, Amphorx, Imagines. Nigra ligno molliori ad utenfilia varia, ad manubria conducit. Alba iisdem uribus ut pracedentes infervit. BETULA lignum prabet album, firmum tenax ad Trahas. Rotarum axes, \& varia opera Ruftica ad circulos pro doliis majoribus conftringendis. ALNus 
Lignum molle ad manubria, Vafa Lactis, Coli Rotas, Orbiculos adhibetur. Sub aquis, in folo humido folidillima fundamenta prs. bet, ad palos, Antlias adhibetur. Cum progreffu Temporis fub aqua lapidelcit, SALIX alba, caprea ftipitibus \& perticis fervit. Olim fcutis ob lentorem inferviebat, cultris acuendis adhibetur. Viminum ufus ad Corbes, Fifcinas, Clathros $\&$ alia utenfilia. $\mathrm{S}_{\mathrm{A}}$ LIX purpurea, viminalis Virgis tenaciffimis laudatur. FRAXINUS excelfior commendatur ligno tenaci, duro, obedientiffimo in omni opere ad Ephippia, Aratra, Rotarum Canthos, Rhedas, Vehicu1a. Scalas, Menfas, Cochlearia, Pocula, Manubria, doliorum Circulos. A CER PJeudo Platanus ligno lavi, albo Tornatorum operibus convenit. Acer campeftre imprimis annofa undatim crifpatur \& lautiori fuppellectili, orbibas, menfis adhibetur. Cornus foemina ligno duro, teriaciflimo gaudet. E. NO N M U v vulgaris preItat ligno tenero folido, fifili lutefcente ad Buxum accedente, RIB ES dentibus pećtinum infervit. HEDERA dat pocula medicata \& lavigandis cultris eft aptum. ULM Us Lignum prabet durum, tenax ferò putrefcens ad prela, axes, Rotas, volvulos linteorum. $\bar{P}_{R}$ u us Spinofa dat lignum durum tenax pro pratorum pectinibus. Spinz obturandis foraminibus cariofi Cadorum ligni adhibentur. PADUs lignum babet lave, flexile ad manubria; rami flagellis \& fiftulis tabacariis conveniunt. $C_{R A T E}$ GUS Aria, Oxy. acantba duro, tenaci, glabro ligno gaudent. SO B Bus aucuparia ad Rotarum radios, temones \& nodofos baculos fervit. PY R US communis ligno duro, compacto, glaberrimo ad Inftrumenta varii generis, Mathematica, fabrorum lignariorum, Tornatoribus \& qui ligno figuras infculpunt impr. expetitum. Malo lignum durius. I $L 1$ \& lignum populneo mollius, glabrumteredini non obinoxium, porofum, ad calopodia, fimulacra, Sutorum menfas expetitum. Cy I s o Laburno Lignum denfum, durum, tenax, Ebeni fuccedaneum ad opera nitidiora. Tibiis quoque infervit \& Rotarum dentibus.. Sed \& elegantioribus, pretiofioribus vafis \& operibus Scriniariis \& Torno elaborandis infervire poffunt ligna varia undatim crifpa circa radices vel in Tophos \& nodos excrefcentia venis undulatis verficoloribus uti Radix \& Tophi crifpi Alni, Betule, Ace- 
ris platanoidis, Campefris, Juglandis, Sorbi aucuparix \& aliarum. Ut taceam Lignorum exoticorum qux, denfitate, nitore, colorum varietate \& mixtura, odoris gratia, Lignorum durabilitate, proceritate pluribus dotibus placere poffunt maximam copiam, qux palfim apud Auctores Rei Herbaria defcribuntur $\&$ in R AII, SLOANEı, BR OVVNII, JACQUINI operibus defcribuntur. Europzarum verò Hiftoriam \& ufus ex Ill. HALLERI (x), LINN更 $1(y)$, HAMELII (z) \& OTTII (a) noftri Scriptis cognofcere licebit,

\section{XI.}

Eft autem Lignorum delectus non modo in ipfa Arboris fpecie Lignonum habendus, fed $\&$ in ejusdem loco natali, xtate, tempore quo delectus im cæditur, præparatione, partibus, fectione, figura \& itructura qua ad Firmita \& firmilfima fit \& cujus partes fe mutuò fulciant : quæ parrim tem. ex principiis Mathefeos non Elementaris modo, fed fublimioris, partim ex principiis Phyficis generalioribus, atque fpecialioribus de Vegetatione Arborum \& Lignorum firmitate ac refiftentia inftitutis experimentis funt dijudicanda. In quo experimentorum genere facile omnibus palmam præripuit Cel. MuSCHENBROECK (b) qui lignorum refiftentiam abfolutam $\&$ relativam in quamplurimis lignorum generibus, in parallelipipedis \& cylindris ligneis machina claftica difcerptis, diffractis, vel incumbente pondere comprimente ruptis exploravit, fimul verò eorum denfitatis vel gravitatis fpecificx accuratam Tabulam exhibuit. Quibus addi poffunt D 3

$\mathrm{Cl}$.

(x) Enumer. Plantar. Helv, Goetting, 1742, f, \$16h. Decon. Befet, fahaft ju Bern, T. IV. 1763. p. 8 .

(y) Flor, Lappon. \& Suec.

(z) Traité \& Phylique des Arbres.

(a) Dendologia Europæ medix, 3itri 1763.8 ,

(b) Diff. Phyficx Exper. \& Geometr. L. Bat, 1729. 4. Introd.ad Philor. Natur, Leidx $17^{52}, \mathrm{p}, 414,483$. 
Cl. PAREN (c), de BUFFON (d), HAMELh (e) fuper hoc argumento lcripta, cum iis qux fapienti felectu habito, \& fuis experimentis tdoctus Vir Spectatiff. \& integerrimus Amicus nòfter D. J. J. Ortrus f) contulit in utili Dendrologix Europx Opufculo. Eft ex Obfervationibus Mufchenbrockianis reffiftentia abfoJuta qua ligna refiftunt potent a: fecundum fibrarum longitudinem trahenti in Tilia \& Alno 1000 in Quercu Ir 50. In Fago \& Fra. xino 1250. in Ulmo 950. in Abiete 600. In Picea 550. Parallelipedum $\frac{27}{100}$ poll. rhenol in machina clattica à totidem libris quot numeri judicant fuit difcerptum.. Cohærentia refpectiva five refi. ftentia quam exercent adverfus potentiam in fibras longitudinales perpendiculariter agentem in lignis iisdem eft in ratione inverfa Kongitudınis, directa fimplici latitudinis \& duplicata altitudinis: Ratio verò ad abfolutam difficulter patefcit \& nonnifi per experimenta cognofcitur ob variam elongationem quam fibræ $a b a p$ plicatis ponderibus patiuntur, \& hinc, ex curvatura momentum mutatur. Fuerunt nempe abfoluta ad refpectivas in Fraxino uti 18. Fago I6. Picea inter I2 \& 14. Quercu \& Ulmo inter I I \& 12. in Tilia inter 10 \& I1. Refiftebant verò ponderibus incumbentibus ferè uti quadrato- quadratum lateris bafeos. Fruftum Tiliæ parallelipipedum cujus bafis quadrata lateris femipollicaris longitudo 4. pedum ab incumbente pondere 5 . librarum diffractum eft Rationes verò reffftentiarum fuerunt hujusmodi: Tiliæ 206. Piceæ 226. Fagi 146. Quercus 86. Ex Obfervationibus $\mathrm{Cl}$ du $\mathrm{HA}_{\mathrm{A}}$ MEL (g) porro conttat ligna imprimis querna duriora effe qux in locis nafcuntur ficcis, perflatis, calidioribus, arborum folitariarum, quam in denfis Sylvis natarum, in arboris parte ficciori, denfiori, radici \& meditullio propiori, quam verfus fummitatem, corticem, ramos. Ef enim Arbor quaf ex Conis fibi incumbentibus tota

(c) Mén. de l'Acad. des Sciences, 1907, 512. 1710, 17\%。

(d) Mém. Sc. 1738.169:1741. 292., 1742.335.

(e) De l'Explication des Bois، à Paris 1764.2. Yol. \&

(f) 1. c. p. 194.

(g) 1. co P, I, Chap, s. p. jo, feqg. 
compofita, quorum intimus breviffimus \& maxime adultus, cxteris durior, reliqui, quo exteriores eo longiores \& juniores iidemque molliores. Experimenta verò Mufchenbroekiana evincere viden = tur, medium quendam effe locum inter corticem \& axem, atque inter radicem \& fummitatem in qua maxima ligni firmitas detur.

Quanam verò fit Trabis ex Cylindro exafcianda ratio altitudinis ad bajin, in qua maximam obtinet refiftentiam, per Calculum differentialem determinatur. Sit Diameter, Arboris $=\mathrm{D}$. latitudo Tra: $\mathrm{bis}=\mathrm{B}$. quxritur altitudo $\mathrm{A}$. Cum hic detur Triangulum rectangulum cujus bafis B. altitudo A. hypothenufa D. Erit ex Theore: mate Pythagorico altitudo $A=D D-B B$. Refiftentix verò funt in ratione compofita fimplici bafeos \& duplicata altitudinis. Ergo Refiftentia Trabis erit $=\mathrm{DD}-\mathrm{BB} \times \mathrm{B} .=\mathrm{DBB}-\mathrm{B}^{3}$. Hac itaque erit Sećtio transverfa tigni onus maximum ferentis. Et ex Natura Quantitatum variabilium erit hujus maximi differentiale, quantitas evanefcens. Ergo $D D \cdot d B-{ }_{3} B B d B=0 . \&$ hinc $D D=3 B B$. quo, valore in priore xquatione pro $\mathrm{A}$ fubftituto erit $\mathrm{A}=\sqrt{3} \mathrm{BB}-\mathrm{BB}=$ $\sqrt{2} \mathrm{BB}$. \& quxfita ratio latitudinis ad altitudinem $\mathrm{B}: \sqrt{2} \mathrm{BB}=\mathrm{I}: \sqrt{2}$. $=I: 414 ;$ ratio qux propius accedit ad rationem $2: 3$. quam $3: 4$ : Itaque feligunt Architecti rationem fesquialteram $I: I \frac{1}{2}=2: 3$. uti legitur apud CJ, KRAFT (b) \& KESTNER. (i)

\section{XII.}

Quando verò Tempeffivum maxime fit Arbores cadere, ut Lig- Tempus num corruptioni magis refiftat, minusve à Termite aliisve In- norum opfectis Ligniperdis lædatur, varia præcipiunt Architecti. Sunt in portunum. his non pauci, qui ex prejudicata opinione Infecta ex putredine generari, \& Plenilunii tempore Arbores fucco maxime abundare opinantes, ftatuunt opportunum maxime crendis Arboribus Tempus hybernum quod in Novilunium vergit, Sed neque

(h) Diff. de Triglyphis , pag. 16.

(i) Hamb, Magazin. T. II. $p, 64 \%$ 
que Phafibus Luna in Vegetabilia affignata poteftas, neque Inlecicrum ex putredine genefis ex Oblervationibus legitimo rasiocinio colligitur. Sane ne quidem Atmofpharæ pondus à Lunx phafibus fenfibilem mutationem pati, qux in Mercurium Tubi Barometrici effectum exhibeat, plufculorum annorum obfervationes docent $k$ ). Neque Animalcula minutiflima, qux in Infufionibus rerum putridarum - per Microfcopium deteguntur, neque plurima Infecta, putridum inhabitantia, idemque ex Sapienti Creatoris inftituto confumentia, neque Vermes varii lignum deftruentes, ut funt Larva variorum Cerambycum, Scarabaorum imprimis Naficornis, Cervi, Pbalene Colli, Tenebrionis molitoris, Blatta lucifugæ \& Orientalis \& plura alia Infecta in his corporibus nafcuntur, fed ex Ovulis hic depofitis demum evolvuntur. Ea enim Naturali inftinctu, Effluviorum dulcedine allecta in his fibi vel foetibusque gratis locis, ovula vel foetus deponunt, quod in his putredine diffolutis partibus commode in apto habitationis, fotus, nutrimenti loco vivere poffint : Prout id Obfervationibus Clar. Virorum REDI, SvVA MERDAMII, REAUMURII \& prxcipuorum Eptomologorum confirmatur. Neque Animalcula Infuforia fine adventitiis ex aëre ovulis nafci ex nuperrimis Clariff. SPALLANZANI (1) aCcuratiffimis experimentis conftat. Cum enim Decoctum fervens variorum Seminum tubis vitreis infundebat, vitro fufo hermetice claudebat, \& in fervente aqua aliquandiu detinebat nulla deinceps animalcula in liquore nata funt, niff fortaffe fiffura $\mathrm{Vi}$ tri aëri aditum paraffet. Corruptionis caufa prxcipua elt, in Ligno copia humidi ftagnantis, quod in vivente Planta per Vafa motum fuas partes intime habet commixtas \& cum Aëre interno fixo, primario Corporum Naturalium vinculo unitas.

$\mathrm{H} \mathfrak{x}$ (k) Lambert in Act: Helvet: T. IV. p. 315. du Hamei 1.c.
1. 380 .

(1) Diff due dell' Abate Spallanzanlo Saggio di Olfervaz. Microfcop. concementi il Siftema della Generazione, in Modena 1755 . 4. Capit, X. p: 78. Seq. 
$\mathrm{H} \mathfrak{x}$ in quiefcente humore viribus incernis propriis liberius oblequentes juxta Affinitatis leges coeunt, alix aliis uniuntur, à priori nexu folvuntur, arctıfima unionis medium Aër halituofus, falfus, inflammabilis erumpit, elafticus recedit, tota mixtio dePtruitur, effervefcentia, fermentatione, putredine novum mixtum producitur. Quanta enim humorum ad putredinem aut fermentationem vergentium copia etiam ficcis lignis infit accuratiffima HALESI I Experimenta definiunt. Invenit namque in experiendo Sagaciflimus \& Reverendus D. HALE (m) in femipollice Cubico Ijgni quercini I35. granorum ponderis, 44. grana humidi inter deftillandum expulli; fuit ergo pars reliqua folida or granorum. Et majorem etiam rationem partium fluidarum ad folidas invenit D. du HAMEL (n) nimirum $7: 15$. Ex his $9 I_{\text {. }}$ granis prodeunt continuata deftillatione 128 . pollices cubici latentis aërismortiferi, qui inclufum pafferem fubitò occidebat \& à flamma candelx incendebatur. Itaque Voluminis ligni, ad volumen aëris reclufi $I: 2,56$. Et pofito pondere pollicis cubici aëris $=\frac{2}{7}$ grani erit ratio ponderis aëris ad pondus folidi in ligno hærentis uti $37 \frac{4}{7}$ ad $53 \frac{3}{7}$ in ratione majore, fubtripla. Quis ergo mirabitur in ea diverfitate partium in hoc mixto quiefcente Vegetabili putredinem facile nafci, eo etiam citius, quo ligno major humidi aquofi copia ineft. Itaque hoc Lignum putredini magis refiftet, quod ex fana Arbore habetur, cujus radices nec vetuftate confumtx, nec Ventorum agitatione labefactatx funt, qux loco ficciori \& perflato nata eft, qux cxditur Eftatis initio vel decurfu, tempeftate in qua plantx magis perfpirant, pauciori fucco turgent \& refectæx citius ficcantur, qux cæaditur poft decorticationem, aut 'cxla citò decorticatur, ut ea ratione Alburnum in Lignum denfetur, Lignique fubftantia denfior reddatur, \& Infecta à Cortice \& Alburno arceantur.

Sed plura de his addere neque Tempus neque DiffertatioE

nis

(in) Statikal Effays, Vol. I, Lond, 1731, Exper. $55.5 \%$

(n) 1. c. T. L pag. 16, 
nis fcopus concedunt. Ea qux hactenus de Arborum \& Planta. rum in Edificiis Sacris \& Civilibus Vitæ Commoditatibus ufu expofita funt fatis docent, quot \& quanta fint munera à benigniffi. mo boni omnis Difpenfatore hominibus conceffa, qua fane Mentem efficacifime excitare poffunt ad plenam in DEUM fiduciam, ad devotam Venerationem atque ad latam fpem immortalium, coeleftium habitationum à Sotere fidelibus paratarum, in Civitate cujus \& Conditor eft ipfe DEUS./Arifex.

(o) Epift, ad Hebr, XI, 10, Apocal, Cap, XX.

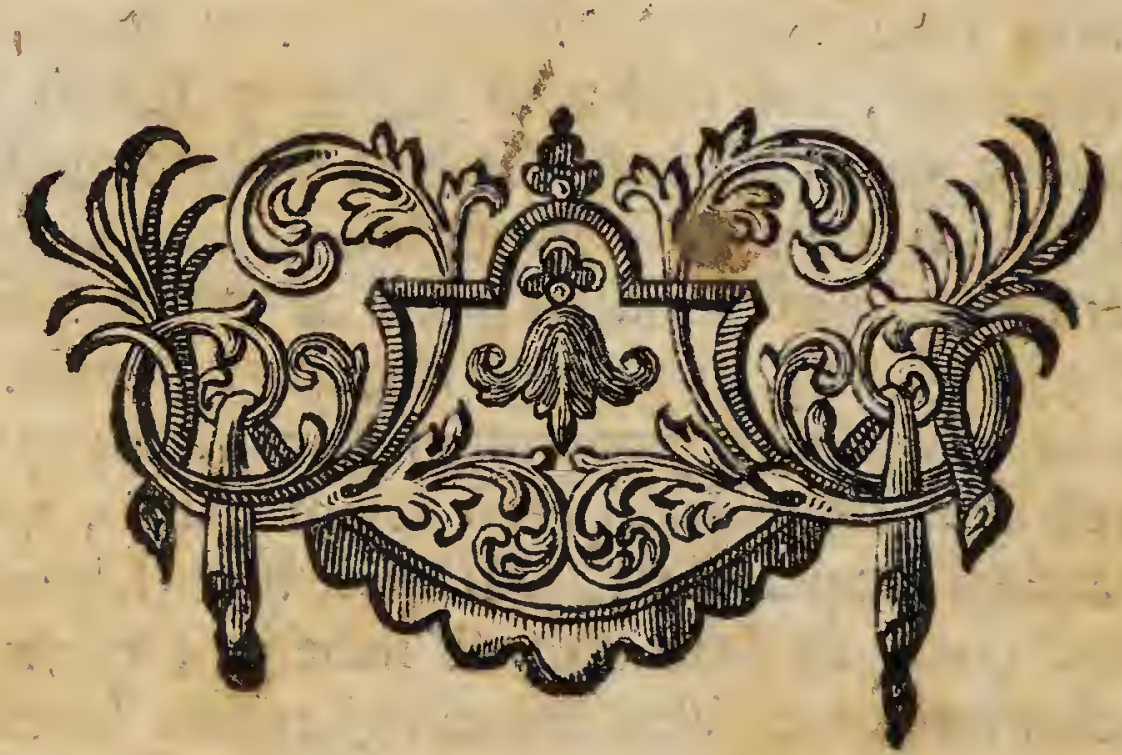

